\title{
Numerical Research in Wall Mass Transfer Characteristics of the Straight Pipe under Fully Developed Turbulent Flow
}

\author{
Jie Li \\ Jingdezhen Ceramic Institute, Xianghu Town, Jingdezhen 333403, Jiang Xi, P.R. China \\ jack197745@gmail.com
}

\begin{abstract}
Keywords: Straight pipe; Fully developed turbulent flow; Wall mass transfer; Numerical simulation Abstract. Numerical simulations were performed to determine the effect of the Reynolds number $(R e)$ on the wall mass transfer rate of the straight pipe under the fully developed turbulent flow. The simulations were conducted for $R e=20,000,40,000$ and 70,000. Because the viscous sublayer is a key zone to the wall mass transfer of the pipe, the low Reynolds Number $k$ - $\varepsilon$ turbulence model was applied, by use of which the mass transfer in the viscous sublayer could be calculated out. The Schmitt number $(S c)$ was determined to be 1280 in the research. On basis of the simulation results, the average Sherwood number $\left(S h_{\mathrm{a}}\right)$ along the pipe axis at $R e=20,000,40,000$ and 70,000 was determined to be 886,1668 and 2835 , respectively, all a little lower than but close to the corresponding values, 1110, 1934 and 3026, which were derived from the correlation $S h=0.0023 R e^{0.8} S c^{0.4}$ [1].
\end{abstract}

\section{Introduction}

Mass transfer enhancement is regarded as one of the elements leading to flow-accelerated corrosion (FAC) in the piping system of the nuclear and fossil power plants. The common pipe components include the elbows, tees, valves, and so on, so researches in mass transfer characteristics of these pipe components are very important. Jinbiao [2] numerically researched about mass transfer enhancement downstream of an orifice, so did El-Gammal [3] and Hwang [4]. Jinbiao investigated effects of Reynolds number, orifice thickness and diameter ratio and investigation results showed that the locations of reattachment point and the peak transfer rate point were not affected by the Reynolds number, and a thin orifice helped mass transfer enhancement in its downstream. Moreover, the peak transfer point appeared at about $0.4 L_{r}$ (reattachment length) downstream of the orifice. On basis of the simulation results, El-Gammal [3] drew conclusions that the Sherwood number (Sh) increased sharply downstream of the orifice, reaching a maximum within 1-2 diameters downstream of the orifice, before relaxing back to the fully developed pipe flow value. The research about the orifice besides, El-Gammal [5] got the surface wear pattern caused by the flow in a 90-degree elbow on basis of experiments and simulations.

Though the research in mass transfer characteristics of straight pipes is a little simpler, it offers a base for the researches about the comliated pipe components. The paper is to numerically research in the wall mass transfer characteristics of the fully developed turbulent pipe. And the objectives of the study are to get $S h$ values at $R e=20,000,40,000$, and 700,00 and then get the relations among $S c, R e$ and $S h$.

\section{Numerical Analysis}

Numerical simulations were performed to get the wall mass transfer rates in a 1-in. diameter $(D)$ and length $L=5 D$ hydrocal $\left(\mathrm{CaSO}_{4} \cdot 2 \mathrm{H}_{2} \mathrm{O}\right)$ pipe at $R e=20,000,40,000$, and 70,000 under fully developed flow. Since the flow is axisymmetric, a two-dimensional model was considered to reduce the computational time.

The governing conservation equations for continuity, momentum and species for incompressible fluid are

$$
\frac{\partial \bar{u}_{i}}{\partial x_{i}}=0
$$




$$
\begin{aligned}
& \bar{u}_{j} \frac{\partial \bar{u}_{i}}{\partial x_{j}}=-\frac{1}{\rho} \frac{\partial \bar{p}}{\partial x_{j}}+\frac{\partial}{\partial x_{j}}\left\{v\left(\frac{\partial \bar{u}_{i}}{\partial x_{j}}+\frac{\partial \bar{u}_{j}}{\partial x_{i}}\right)-\overline{u_{i}^{\prime} u_{j}^{\prime}}\right\} \\
& \bar{u}_{j} \frac{\partial \bar{c}}{\partial x_{j}}=\frac{\partial}{\partial x_{j}}\left\{D_{\mathrm{AB}} \frac{\partial \bar{c}}{\partial x_{j}}-\overline{c^{\prime} u_{j}^{\prime}}\right\}
\end{aligned}
$$

where $\bar{u}_{i}$ and $u_{i}^{\prime}$ are the mean and fluctuating velocity components $(i=1,2), \bar{c}$ and $c^{\prime}$ are the mean and fluctuating molar concentration of the species in the mixture, $v$ is the kinematic viscosity of the fluid, and $D_{\mathrm{AB}}$ is the mass diffusivity between two species in the mixture, which is that between water and hydrocal at $25^{\circ} \mathrm{C}$ in the study and is taken as $6.98 \times 10^{-10}$ as [3].

As for the boundary conditions, the hydrocal concentration at the wall was taken as $2.4 \mathrm{~kg} / \mathrm{m}^{3}$, the saturation concentration of the hydrocal in the water, taken as 0 at the axis. The outlet of the research domain was set as the pressure-outlet at pressure $p=0 \mathrm{~Pa}$.

The GAMBIT code and FLUENT code were respectively used to generate the computational mesh and conduct the simulation. In order that mass transfer in the viscosity sublayer could be solved, the low Reynolds Number $k$ - $\varepsilon$ turbulence model was used and so the maximum $y^{+}$value for the first cell close to the wall should not exceed 0.125 [3]. Corresponding to the $y^{+}$value of the first cell close to the wall, its $y$ value could be determined. The definition formula of $y^{+}$is

$$
y^{+}=\frac{y u_{*}}{v}
$$

where the friction velocity $u_{*}$ is defined by the formula

$$
u_{*}=\left(\tau_{w} / \rho\right)^{\frac{1}{2}}
$$

The Reynolds stress at the wall, $\tau_{w}$, is estimated from Fanning equation

$$
\tau_{w}=\frac{f \rho v^{2}}{8}
$$

where $v$ is the velocity value and $f$ is the friction factor. By checking the Moody's figure, $f$ was taken as 0.019 about for flow in the smooth circular pipe at $R e=70000$.

By combining the formulas (4)-(6), it was obtained

$$
y=\frac{y^{+} v}{\left(\frac{f}{8}\right)^{\frac{1}{2}} v}
$$

where the $v$ value of water at $25^{\circ} \mathrm{C}$ is $8.94 \times 10^{-7} \mathrm{~m}^{2} / \mathrm{s}$. From the formula $R_{e}=\frac{v D}{v}$, the $v$ value was derived to be $2.46 \mathrm{~m} / \mathrm{s}$ under conditions of $R e=70000$ and $D=1$ in.. By putting the $y^{+}$value of 0.125 , and the corresponding values of $f, v$, and $v$ into formula (7), the $y$ value for the first cell close to the wall was obtained to be $y \leq 0.0009 \mathrm{~mm}$.

Because the length of $0.0009 \mathrm{~mm}$ is very short, the block-structured mesh was used, the whole computational zone being divided into 16 zones with different grid sizes. The radial length and axial length were set as $y$ and $x$ coordinate respectively in GAMBIT. From the wall to axis the wide of the grids, $\Delta y$, increased and the length of the grids, $\Delta x$, also increased when the zone changed, but $\Delta x$ kept the same in a zone. As a principle, the increase ratio for the adjacent grids should not exceed 1.2 and the aspects of all grids should not exceed 5. The sum of the grids in the whole computational domain was about 540,000 and a partial mesh graph is presented as fig. 1 . The convergence criterion applied to the residuals of the main flow and mass transfer parameters was less than $10^{-8}$. 


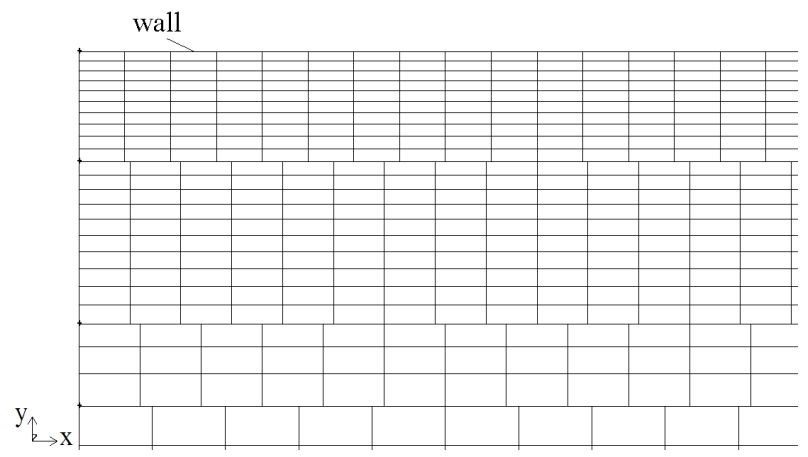

Fig. 1 a part of the mesh graph

The study researches in wall mass transfer of a fully developed turbulent flow pipe, so in order to get the $v, k$, and $\varepsilon$ profiles the flow in a $L=50 D$ pipe was simulated before the simulation of mass transfer. For the same cause, the maximum $y^{+}$value for the first cell close to the wall could not exceed 1 when the flow simulation was conducted. And the corresponding $y$ value was derived to be $0.007 \mathrm{~mm}$. Similarly, the block-structured mesh was used and the whole computational domain was divided into 10 zones. The inlet of the research domain was set as velocity-inlet and the outlet as pressure-outlet at $p=0 \mathrm{~Pa}$. The convergence criterion was taken as the same as that in the mass transfer simulation. The $v-y(r), k-y$, and $\varepsilon-y$ profiles at the outlet cross section coincide with those at the cross section of $L=45 D$ and the coincidence of the $v-y$ profiles is shown in Fig. 2. The coincidence of the profiles proves that the flow has fully developed at the outlet.

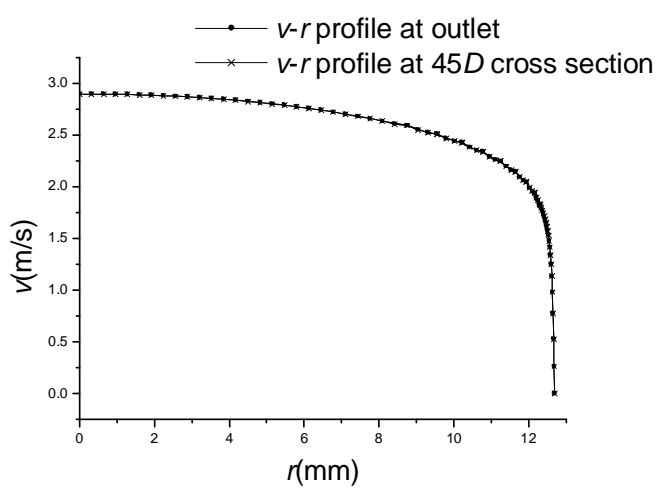

Fig. $2 v-r$ profiles at outlet and $45 D$ cross section from flow simulation

The $v-y, k-y$, and $\varepsilon-y$ profiles at the outlet gotten by the flow simulation were written into the inlet boundary conditions for the mass transfer simulation in form of profile files and then the mass transfer simulation was conducted.

The $v-y, k-y$, and $\varepsilon-y$ profiles at the outlet cross section all coincide or nearly coincide with those at the inlet and the coincidence of the $v-y$ profiles is shown in Fig. 3.

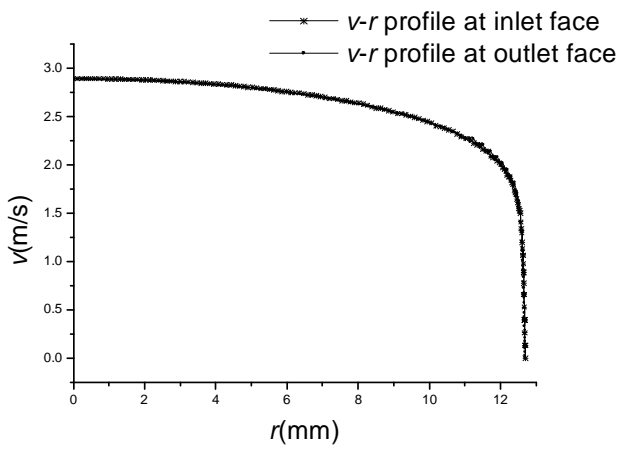

Fig. $3 v-r$ profiles at inlet and outlet face from mass transfer simulation

It is derived by calculation that the mesh for mass transfer at $R e=70,000$ is appropriate for those at $R e=20,000$ and 40,000. 


\section{Results and Discussion}

On basis of the mass transfer simulaiton results, the average Sherwood number along the $x$ coordinate, $S h_{\mathrm{a}}$, could be derived. Take the whole pipe as the research domain, denoted as $\Omega$. The mass flow rate of the hydrocal from the wall into $\Omega$, $h$, was calculated by the formula

$$
n=A_{\mathrm{w}} \rho h_{\mathrm{a}}\left(w_{\mathrm{w}}-w_{\infty}\right)=\pi D L \rho h_{\mathrm{a}}\left(w_{\mathrm{w}}-w_{\infty}\right)=\pi D L \rho h_{\mathrm{a}} w_{\mathrm{w}}
$$

where $h_{\mathrm{a}}$ is the average mass transfer coefficient in $\mathrm{m} / \mathrm{s}, A_{\mathrm{w}}$ is the annular wall area of the pipe, $\rho$ is the density of the mixture, and $w_{\mathrm{w}}$ and $w_{\infty}$ are respectively the hydrocal concentration at the wall and in the mainstream area in $\mathrm{kg} / \mathrm{m}^{3}$.

Meanwhile, the net mass flow rate of the hydrocal out of $\Omega$ through the inlet and outlet face, denoted as $n$, was calculated by the formula

$$
n=h\left(w_{\mathrm{o}}-w_{\mathrm{i}}\right)=\rho A v\left(w_{\mathrm{o}}-w_{\mathrm{i}}\right)=\frac{1}{4} \pi D^{2} \rho v\left(w_{\mathrm{o}}-w_{\mathrm{i}}\right)
$$

where $n$ is the mass flow rate of the mixture from the inlet face into $\Omega$ in $\mathrm{kg} / \mathrm{s}$, and $w_{\mathrm{i}}$ and $w_{\mathrm{o}}$ are respectively the mass-weighted average concentration of the hydrocal in the inlet face and outlet face.

According to the law of mass conservation must equal $h$, so the formula solving $h_{\mathrm{a}}$ was gotten

$$
h_{\mathrm{a}}=\frac{\frac{1}{4} D v\left(w_{\mathrm{o}}-w_{\mathrm{i}}\right)}{L w_{\mathrm{w}}}
$$

The definition formula of $S h$ is $S h=\frac{h D}{\mathrm{v}}$ and $S h_{\mathrm{a}}$ is $S h_{\mathrm{a}}=\frac{h_{\mathrm{a}} D}{\mathrm{v}}$, and the $S h_{\mathrm{a}}$ values for mass transfer at $\operatorname{Re}=20,000,40,000$ and 70,000 were gotten to be 886,1668 and 2835 , respectively. The Schmidt number is defined by the formula $S c=\frac{v}{D_{\mathrm{AB}}}$ and its value equals 1280 in the study.

Meantime, the $S h$ values for wall mass transfer at $R e=20,000,40,000$ and 70,000 in a circular pipe under fully developed flow were gotten to be 1110, 1934 and 3026 by use of the widely accepted correlation $S h=0.0023 R e^{0.8} S c^{0.4}$ [1]. The $S h_{\mathrm{a}}$ values derived from the simulation are a little lower than but close to those gotten by the correlation above.

\section{Conclusions}

The $S h$ values for wall mass transfer at $S c=1280$ and $R e=20,000,40,000$ and 70,000 in a circular pipe under fully developed flow are gotten to be 886, 1668 and 2835 in the study. The data are a little lower than but close to the values, 1110, 1934 and 3026, which are gotten from the widely accepted correlation $S h=0.0023 R e^{0.8} S c^{0.4}$.

\section{References}

[1] Y.A. Çengel, Heat and Mass Transfer, third ed., McGraw-Hill Education, New York, 2007.

[2] J. Xiong, X. Cheng, Y. Yang, Numerical Investigation on Mass Transfer Enhancement Downstream of an Orifice, I. J. of Heat and Mass Transfer, 68 (2014) 366-374.

[3] M. El-Gammal, W. H. Ahmed, C. Y. Ching, Investigation of Wall Mass Transfer Characteristics Downstream of an Orifice, Nuclear Engineering and Design, 242 (2012) 353-360.

[4] K.M. Hwang, T.E. Jin, Identification of the Relationship between Local Velocity Components and Local Wall Thinning inside Carbon Steel Piping, J. of Nuclear Science and Technology 46 (2009) 469-478 
[5] M. El-Gammal, H. Mazhar, J.C. Cotton, and etc., The Hydrodynamics Effects of Single-phase Flow on Flow Accelerated Corrosion in a 90-degree Elbow, Nuclear Engineering and Design, 240 (2010) 1589-1598. 